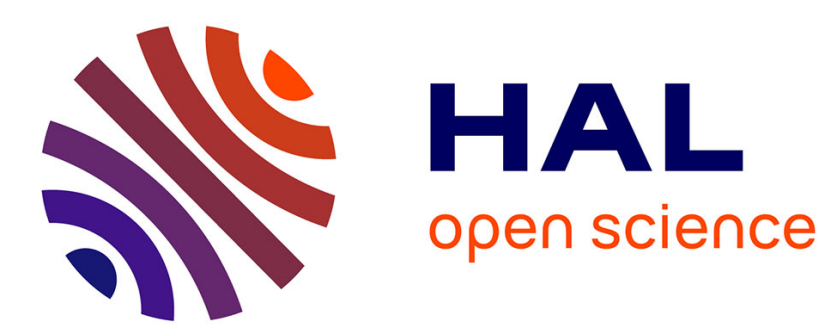

\title{
Mettre le feu aux poudres ou passer inaperçu? Stratégies de résistance à Lampedusa et à Calais \\ Camille Guenebeaud, Annalisa Lendaro
}

\section{To cite this version:}

Camille Guenebeaud, Annalisa Lendaro. Mettre le feu aux poudres ou passer inaperçu? Stratégies de résistance à Lampedusa et à Calais. Cultures \& conflits, 2020, 117, pp.79-96. 10.4000/conflits.21541 . halshs-03090899

\section{HAL Id: halshs-03090899 \\ https://shs.hal.science/halshs-03090899}

Submitted on 30 Dec 2020

HAL is a multi-disciplinary open access archive for the deposit and dissemination of scientific research documents, whether they are published or not. The documents may come from teaching and research institutions in France or abroad, or from public or private research centers.
L'archive ouverte pluridisciplinaire HAL, est destinée au dépôt et à la diffusion de documents scientifiques de niveau recherche, publiés ou non, émanant des établissements d'enseignement et de recherche français ou étrangers, des laboratoires publics ou privés. 


\section{Mettre le feu aux poudres ou passer inaperçu ? Stratégies de résistance à Lampedusa et à Calais}

Résumé : L'île italienne de Lampedusa et la ville de Calais sont devenues des symboles de la frontière contemporaine. Régulièrement traversés par des « crises », ces territoires et leurs populations sont gouvernés au travers de dispositifs de contrôle et de tri qui questionnent l'effectivité de l'état de droit. Malgré ces similitudes, Lampedusa et Calais se distinguent par leur position géostratégique dans la gestion du phénomène migratoire et par des configurations d'acteurs locaux différentes. L'objectif de cette contribution est double : d'une part, il s'agira de montrer que ces spécificités ont des effets sur les stratégies de résistance des personnes en migrations ; d'autre part, il sera question de mettre l'accent sur le caractère adaptatif de ces résistances par le corps : dans quelles circonstances a-t-on intérêt à subvertir l'ordre public et ainsi attirer l'attention des institutions ? Quand est-ce que, au contraire, il vaut mieux mettre en œuvre des stratégies plus discrètes de passing ? Cet article, qui s'appuie sur deux enquêtes qualitatives réalisées par les deux auteur-e-s entre 2013 et 2018, se propose d'apporter des éléments de réponse à ces questions.

Abstract : D’avance merci à Cultures et Conflits pour la traduction du résumé.

Mots clés : Lampedusa, Calais, politiques migratoires, résistances par le corps, XXI siècle.

Keywords : Lampedusa, Calais, Migration policies, Body Resistances, XXI century.

\section{Courte biographie des auteur-e-s :}

Camille Guenebaud est docteur en géographie, chercheur associé au laboratoire Analyse Comparée des Pouvoirs à l'Université Paris-Est Marne-la-Vallée. Par le biais d'une enquête ethnographique sur la ville de Calais, il a étudié les processus d'immobilisation des personnes migrantes et leurs modalités de résistance, ainsi que les pratiques et les discours des acteurs locaux (municipalité, riverains, commerçants) qui, combinés, déplacent la frontière politique entre deux États vers l'émergence de frontières sociales au sein de la ville. Il est membre de l'équipe DisPow (ANR 2019-2021).

Annalisa Lendaro est chargée de recherche en sociologie politique au CNRS (France). Ses principaux intérêts portent sur les politiques migratoires, leurs applications, et les conséquences qu'elles ont sur l'accès aux droits. Elle coordonne l'ANR DisPow (2019-2021), un projet collectif qui se propose d'étudier les formes et les effets du pouvoir discrétionnaire de l'Etat à trois frontières : le Calaisis ; la frontière franco-italienne; et la frontière basque. 


\section{Bibliographie :}

Carens J. (2013), The ethics of immigration, Oxford University Press.

De Genova N. (2018), The "migrant crisis" as racial crisis: do Black Lives Matter in Europe?, Ethnic and Racial Studies, 41:10, 1765-1782.

De Genova, N., \& Peutz, N., eds., 2010, The deportation regime: Sovereignty, space, and the freedom of movement. Duke University Press, Durham, NC.

Guénif-Souilamas N., « Le corps-frontière, traces et trajets postcoloniaux » dans Ruptures postcoloniales, Paris, La Découverte, 2010, p. 217-229

Huysmans J., The politics of insecurity. Fear, Migration and Asylum in the EU, London, Routledge, 2006

Isin, E. F., \& Rygiel, K., 2007, ‘Abject spaces: frontiers, zones and camps’, in Dauphinee, E., Masters, C. (eds.), The logics of Biopower and the War on Terror: Living, Dying, Surviving, Palgrave Macmillan, Basingstoke, 181-203

Isin, Engin Fahri. Being political: genealogies of citizenship. U of Minnesota Press, 2002.

Lazaridis G., 2016. Security, Insecurity and Migration in Europe, London, Routledge

Lendaro A., Rodier C., Vertongen Y. L. (dir.), La crise de l'accueil. Migrations, droits, résistances, Paris, La Découverte, 2019

Rygiel K. (2011) Bordering solidarities: migrant activism and the politics of movement and camps at Calais, Citizenship Studies, 15:1, 1-19 


\section{Gouverner la frontière et lui résister par le corps}

En dépit de la durée et de l'ampleur de la crise de l'accueil qui traverse l'Europe ${ }^{1}$, les politiques européennes contemporaines témoignent de la volonté renouvelée de lutter contre l'immigration irrégulière, plutôt que de garantir l'accès aux droits fondamentaux des personnes en situation de migration. Une vaste littérature documente les effets de cette orientation sécuritaire dans la gestion des frontières internes et externes de l'Union européenne ${ }^{2}$. Situées aux extrémités de l'Espace Schengen, respectivement ville la plus proche des côtes anglaises et île la plus méridionale de l'Italie, Calais et Lampedusa sont les symboles depuis plus de vingt ans de cette gestion répressive des mobilités humaines.

Nous considérons ici la frontière dans sa dimension biopolitique ${ }^{3}$, c'est à dire comme un outil de gouvernance des populations qui passe par leur catégorisation et par leur séparation spatiale, et qui implique l'exercice de pouvoirs sur le corps et la vie des personnes ciblées. Parler de frontière biopolitique revient à s'intéresser aux origines, au fonctionnement, et aux effets des politiques et des pratiques de distinction, de hiérarchisation, et de contrôle sur la vie des personnes migrantes. La nature biopolitique du régime contemporain des frontières européennes se manifeste par des dispositifs qui bloquent, expulsent et trient entre des corps libres de circuler et des corps qui se heurtent à la matérialité des contrôles ${ }^{4}$. Un ensemble de moyens (juridiques, techniques, humains, etc.) opérationnalisent cette gestion de la limitation de la mobilité des « indésirables $»^{5}$ : des technologies d'identification (tests osseux, enregistrement des empreintes digitales, photo-signalement), de surveillance (caméras, drones, systèmes de détection de mouvements, etc.) et d'enfermement (hotspots,

\footnotetext{
${ }^{1}$ Lendaro A., Rodier C., Vertongen Y. L. (dir.), La crise de l'accueil. Migrations, droits, résistances, Paris, La Découverte, 2019 ; De Genova N., "The "migrant crisis" as racial crisis: do Black Lives Matter in Europe? », Ethnic and Racial Studies, vol. 41, n 10, 2018, p. 1765-1782.

${ }^{2}$ Huysmans J., The politics of insecurity. Fear, Migration and Asylum in the EU, London, Routledge, 2006 ; Ceccorulli M. et Labanca N. (Ed.), The EU, migration and the politics of administrative detention, Routledge, London, 2014 ; De Genova N. et Peutz N. (Ed.), The deportation regime: Sovereignty, space, and the freedom of movement, Duke University Press, Durham, NC., 2010 ; Bosworth M. et Turnbull S., «Immigration detention, punishment, and the criminalization of migration », in Pickering S. et Hamm J. (Ed.), The Routledge handbook on crime and international migration, Routledge, London, 2014; Aas K.F. et Gundhus H.O.I., "Policing humanitarian borderlands: Frontex, human rights and the precariousness of life », British Journal of Criminology, vol. 55, n 1, 2015, p. 1-18; Carens, J. H., « The rights of irregular migrants », Ethics \& International Affairs, vol. 22, n 2, 2008, p. 163-86; Houtum H. van \& Boedeltje F., "Europe's shame, Death at the borders of the EU », Antipode, vol. 41, n 2, 2009, p. 226-30 ; Lazaridis G., Security, Insecurity and Migration in Europe, London, Routledge, 2016.

${ }^{3}$ Foucault M., Naissance de la biopolitique: cours au Collège de France (1978-1979), Paris, Seuil, 2004.

${ }^{4}$ Guénif-Souilamas N., «Le corps-frontière, traces et trajets postcoloniaux» dans Ruptures postcoloniales, Paris, La Découverte, 2010, p. 217-229

${ }^{5}$ Agier A., Gérer les indésirables, Paris, Flammarion, 2008.
} 
centres de rétention administrative), mais également des mesures plus informelles de dissuasion et de harcèlement policier qui œuvrent à rendre compliquée l'installation voire la survie des personnes migrantes.

Le corps et son identification sont centraux dans le fonctionnement de la frontière biopolitique, qu'il s'agisse des contrôles au faciès lors des interpellations, de l'identification policière par les empreintes en vue de l'expulsion, ou de l'épuisement par le harcèlement policier. Si le contrôle s'attache aux corps, c'est également par le corps que se mettent en place certaines formes de résistance. Brûler ses empreintes, faire la grève de la faim, s'automutiler, transformer son apparence pour passer pour européen : les personnes migrantes mettent en jeu leurs corps de multiples façons dans la tentative de passer les frontières.

Cet article met au centre de la focale la capacité d'action et de résistance de ces personnes, y compris lorsqu'elles agissent dans des contextes caractérisés par une privation extrême de droits fondamentaux ${ }^{6}$. Il se propose d'analyser en particulier la dimension corporelle des résistances des personnes migrantes dans les deux contextes frontaliers que sont Lampedusa et Calais.

La dimension comparative de l'analyse est centrale dans la mesure où nous faisons l'hypothèse du caractère adaptatif de ces résistances, selon les contextes socio-politiques et spatiaux au sein desquels elles prennent forme. Il s'agira de comprendre comment ces spécificités influencent des stratégies très variées de résistances des personnes migrantes, allant des plus visibles, tels les actes visant à subvertir l'ordre public, aux plus discrètes, impliquant la transformation de son identité. Inspiré du travail de James C. Scott sur 1'infrapolitique ${ }^{7}$, cette contribution se propose de considérer les liens qui existent entre pratiques de résistance invisibles et anonymes, et les formes les plus violentes de révolte qui visent à subvertir l'ordre public.

La première partie de l'article introduit Lampedusa et Calais en les inscrivant dans le paysage plus large des politiques migratoires contemporaines. L'article présente ainsi à la fois leurs similitudes et leurs spécificités dans la gestion des mobilités humaines. La seconde partie éclaire la dimension corporelle des résistances migrantes dans les deux études de cas, tout en insistant sur le caractère

\footnotetext{
${ }^{6}$ Isin E. F. et Rygiel K., « Abject spaces: frontiers, zones and camps », in Dauphinee E., Masters C. (Ed.), The logics of Biopower and the War on Terror: Living, Dying, Surviving, Palgrave Macmillan, Basingstoke, 2007, p. 181-203 ; Clochard O., « Révoltes, protestations et 'résistances du quotidien'. Des étrangers à l'épreuve de la détention », Migrations Société, vol. 164, $\mathrm{n}^{\circ}$ 2, 2016, pp. 57-72 ; Lendaro A., « Investir la rue alors qu'on n'en a pas le droit. L'improbable mobilisation des demandeurs d'asile à Lampedusa (Italie) », Sociologie et Société, XLVII(1), 2015, p. 123-46.

${ }^{7}$ Scott J. C., Domination and the arts of resistance: Hidden transcripts. Yale University Press, New Haven, CT, 2010.
} 
adaptatif de l'agency des personnes en migration. La contribution conclut avec une réflexion sur l'incompatibilité entre l'état de droit et le fonctionnement contemporain de la frontière biopolitique.

\section{Deux configurations socio-spatiales du contrôle : convergences et spécificités}

Lampedusa et Calais sont deux espaces pris dans un contexte commun de renforcement des contrôles aux frontières extérieures de l'Espace Schengen depuis les années 1990, mais qui présentent respectivement des singularités géographiques, juridiques, politiques et sociales. Analyser ce contexte commun, mais aussi les configurations particulières du contrôle dans chacun de ces espaces, sert de premier jalon dans l'analyse des pratiques adaptatives de résistances des personnes migrantes.

L'expression « frontiérisation », empruntée à Paolo Cuttitta ${ }^{8}$, évoque le processus politique qui conduit un territoire à fonctionner en tant que frontière. Elle permet de dénaturaliser cette dernière, en la considérant comme le produit de décisions politiques et de mesures juridiques, et comme un outil de gestion différentiée des populations. Calais et Lampedusa ont connu des processus de frontiérisation qui présentent un certain nombre de similarités, du fait d'un contexte commun de restrictions des migrations au niveau européen.

Si les frontières internes de l'UE sont devenues plus poreuses, voire ouvertes pour la population européenne à la suite de l'entrée en vigueur de la convention de Schengen en 1995, de manière complémentaire, celles qui délimitent le périmètre externe de l'Union se sont refermées brusquement pour un ensemble de ressortissants de pays tiers soumis à l'obligation d'obtenir un visa Schengen préalablement à leur entrée sur le territoire. L'instauration de la politique de visa est centrale dans le processus de frontiérisation qui nous intéresse ici : en tant que stratégie « ban-optique » ${ }^{9}$, elle permet de contrôler les étrangers en amont de leur arrivée à la frontière, mais elle constitue également la cause principale de l'augmentation des entrées irrégulières en Europe. En effet, les tentatives de rejoindre l'Europe par terre et par mer n'ont pas cessé à la suite de l'entrée en vigueur de ces mesures.

A Lampedusa, le premier centre pour migrants est construit en 1996, un an après l'entrée en vigueur des accords Schengen. Ce choix est déterminant pour comprendre le rôle contemporain joué par cette île méditerranéenne dans les processus migratoires sud-nord. Depuis l'ouverture de ce centre, l'île, de

\footnotetext{
${ }^{8}$ Cuttitta P., « La « frontiérisation » de Lampedusa, comment se construit une frontière », L'Espace Politique [En ligne], vol. $25, \mathrm{n}^{\circ}$, mis en ligne le 08 avril 2015, URL : http://journals.openedition.org/espacepolitique/3336.

${ }^{9}$ Guild E. et Bigo D., «1)Le visa Schengen : expression d'une stratégie de «police » à distance », Cultures \& Conflits [En ligne], $\mathrm{n}^{\circ}$ 49, printemps 2003, mis en ligne le 29 septembre 2003, URL : http://journals.openedition.org/conflits/924.
} 
par l'exiguïté de son territoire et l'éloignement de l'Italie continentale, remplit la fonction de concentration et de confinement des nouveaux arrivés. Ce sont les politiques de contrôles migratoires à l'échelle européenne, l'évolution des relations avec les pays nord-africains, et les choix de gouvernements italiens successifs qui en ont fait un territoire aménagé et utilisé pour contrôler, concentrer, trier, transférer les nouveaux arrivants depuis la rive sud de la Méditerranée.

Tout comme Lampedusa, la ville de Calais s'est construite avant tout comme lieu de frontière du fait $\mathrm{du}$ contexte politique européen. Alors que de nombreux États signent les accords Schengen, l'Angleterre reste en dehors de ce processus et la frontière franco-britannique devient une frontière extérieure de l'Espace Schengen. Or, ce ne sont pas les contrôles depuis l'Angleterre qui vont rapidement être au cœur des enjeux, mais bien les personnes migrantes qui tentent de traverser outreManche depuis la France. Afin d'empêcher ces passages, l'Angleterre va pousser à la signature d'une série d'accords et de protocoles pour renforcer les contrôles et les délocaliser en partie sur le territoire français. Au fil des années se forment alors des camps de personnes migrantes bloquées sur le littoral et en particulier dans la ville de Calais.

La frontiérisation de ces deux espaces est marquée par ces politiques de filtrage et de blocage qui contraignent les mobilités et précarisent la vie des personnes migrantes. La mise en place de ces politiques s'est accompagnée d'une augmentation des personnes blessées et des morts aux frontières ${ }^{10}$. Malgré ces processus de frontiérisation convergents liés à un même contexte européen, des différences fortes subsistent entre Lampedusa, île symbole de la frontière euro-africaine, et Calais, ville-frontière entre deux pays du Nord. Ces différences sont liées à leurs statuts et rôles différenciés dans le dispositif politique de gestion des migrations, ainsi qu'à des configurations spécifiques d'acteurs locaux.

A Calais, les personnes migrantes se heurtent à une double contrainte à leur mobilité. D'un côté, l'intensification des contrôles orchestrée par les accords franco-britanniques depuis le début des années 2000 complexifie et rend plus dangereux leur passage outre-Manche. De l'autre, elles font face à des politiques d'expulsion et d'éloignement du nord de la France. En effet, si le gouvernement français

\footnotetext{
${ }^{10}$ A titre d'exemple, la panne d'une embarcation transportant 545 migrants d'origine africaine et la lenteur des secours des Garde-côtes provoquent la mort de 368 d'entre eux le 3 octobre 2013, un des naufrages les plus meurtriers de tous les temps à seulement quelques centaines de mètres de la côte sud-ouest de Lampedusa. Pour une estimation du nombre de décès depuis $2014:$ https://missingmigrants.iom.int/, et http://www.borderdeaths.org/?page_id=7 ; pour aller plus loin au sujet de leur recensement : Heller C. et Pécoud A., «Compter les morts aux frontières : des contre-statistiques de la société civile à la récupération (inter)gouvernementale », Revue européenne des migrations internationales, vol. $33, \mathrm{n}^{\circ} 2$ et 3 , 2017, consulté le 10 janvier 2019. URL : http://journals.openedition.org/remi/8732; Last T. et al., « Deaths at the Borders Database: evidence of deceased migrants' bodies found along the southern external borders of the European Union ", Journal of Ethnic and Migration Studies, vol. 43, n 5, p. 693-712.
} 
assiste les pouvoirs publics britanniques dans les contrôles frontaliers, il applique parallèlement des stratégies qui visent à soustraire les migrants à l'espace public calaisien. Ces stratégies prennent la forme d'expulsions vers les pays d'origine ou de transit, mais aussi de transferts vers d'autres villes françaises via des procédures d'enfermement et d'hébergement. Dès 2002 et pendant plusieurs années, les opérations policières Ulysse organisent ainsi le transport en bus de migrants présents à Calais vers des CADA (Centres d'Accueil pour Demandeurs d'Asile), CHRS (Centres d'Hébergement et de Réinsertion sociale) ou hôtels situés dans d'autres régions françaises ${ }^{11}$. Ces pratiques d'éloignement direct sont secondées par des stratégies policières d'éloignement indirect qui consistent à dissuader les personnes migrantes de rester. Ces mesures «d'auto-expulsion »" ${ }^{12}$ sont très présentes à Calais et se matérialisent par des destructions régulières des campements et des affaires personnelles, des interpellations à répétition, des restrictions d'accès à l'eau et à la nourriture ou encore un empêchement du sommeil ${ }^{13}$. Il en résulte que le fonctionnement de cette frontière biopolitique se caractérise par une double injonction adressée aux migrants : une impossibilité de traverser, mais également une impossibilité de rester en France.

A Lampedusa, le passage de la frontière a déjà eu lieu, l'île n'est qu'un espace de transit pour les personnes migrantes qui attendent leur transfert vers l'Italie continentale. Personne ne reste ni ne souhaite rester à Lampedusa : le transfert est la seule option possible, y compris lorsqu'un rapatriement vers le pays d'origine ou un pays de transit est prévu. Ces transferts s'organisent actuellement via le hotspot construit en 2007 au fond d'un vallon encaissé, loin des regards. Le lieu est régulièrement surpeuplé, et les personnes y sont retenues au-delà de la durée maximale fixée par la loi, dans des conditions qui ne respectent pas les standards nationaux et internationaux ${ }^{14}$, sans être informées systématiquement et en présence d'un interprète des différentes procédures les concernant. De plus, elles sont dans l'impossibilité presque systématique de saisir la justice italienne ou européenne de par l'absence d'experts juridiques et notamment d'avocats exerçant sur place. ${ }^{15}$ Dans ce contexte, afin

\footnotetext{
${ }^{11}$ Clochard C., Le jeu des frontières dans l'accès au statut de réfugié - Une géographie des politiques européennes d'asile et d'immigration, Université de Poitiers, 2007, p. 293.

${ }^{12}$ Fassin E., Fouteau C., Guichard S. et Windels A., Roms et riverains : une politique municipale de la race, Paris, La Fabrique, 2014.

${ }^{13}$ Guenebeaud C., «Le corps face à la frontière. Étude de la répression des migrants sans-papiers à la frontière francobritannique. », Corps, Editions CNRS, n¹4, 2016, pp. 31-39.

${ }^{14}$ Garante Nazionale dei diritti delle persone detenute o private della libertà personale, Relazione al Parlamento, 2018, online : https://www.osservatoriodiritti.it/wp-content/uploads/2018/06/hotspot-migranti.pdf ; Amnesty International, Hotspot Italia, 2016, online : https:/www.amnesty.org/download/Documents/EUR3050042016ITALIAN.PDF

${ }^{15}$ CILD, Hotspot e Centri di Permanenza per i Rimpatri, Violazioni dei diritti umani e dei diritti di difesa dei migranti, online : https://cild.eu/wp-content/uploads/2018/04/Dossier-Lampedusa.pdf
} 
d'éviter les tensions et les actes violents, les forces de l'ordre permettent officieusement aux migrants de sortir et de rentrer dans le centre à travers des passages qu'eux-mêmes se sont ouverts dans les grillages qui les séparent de l'extérieur.

De ce fait, à Lampedusa, une certaine souplesse dans sa gestion permet aux migrants une présence dans l'espace public. Au niveau des contrôles d'identité, tout se joue lors de la prise en charge au sein $\mathrm{du}$ hotspot, et ce n'est donc pas dans les rues et les places du village, sur ses plages, qu'ils sont effectués. Aucun contrôle policier n'a lieu dans l'espace public : paradoxalement, puisque l'île même est une prison en plein air de par son éloignement et l'impossibilité de la quitter par ses propres moyens, leur présence en dehors du centre de rétention n'est pas contrôlée, entravée ou sanctionnée. A l'inverse, à Calais, les personnes ne sont pas confinées dans un seul espace d'identification et de rétention, et les contrôles se matérialisent dans la ville. ${ }^{16}$ Parcs, places, bancs : tous les lieux de stationnement possibles des migrants dans l'espace public font l'objet d'un potentiel contrôle policier dans le but de leur faire quitter la ville.

La maire Les Républicains Natascha Bouchart, qui a succédé en 2008 à trente ans de municipalité communiste, s'est distinguée dans son deuxième mandat par sa volonté de fermer certains équipements publics aux personnes migrantes. L'équipe municipale a fait modifier le règlement intérieur de la médiathèque en 2014 pour ne plus rendre accessible l'espace multimédia et l'accès à internet qu'aux personnes qui disposent d'un document d'identité et d'un justificatif de domicile. La même procédure a été utilisée en novembre 2015 pour restreindre l'accès à la piscine Icéo. Les migrants sont explicitement visés. Cette politique municipale qui travaille à disperser et invisibiliser les personnes migrantes dans la ville est renforcée par l'action de commerçants qui refusent l'accès de leur établissement aux personnes qu'ils identifient comme migrantes ${ }^{17}$. La volonté de certains commerçants d'interdire l'accès à leurs locaux ou à leurs services se retrouve également à Lampedusa. En 2011, un arrêté municipal signé par le maire de l'époque, Bernardino De Rubeis, a officialisé l'interdiction de vendre de l'alcool aux migrants en attente de transfert, et par la suite des nombreux commerçants ont refusé de le faire même si les deux conseils municipaux successifs n'ont pas voté pour le renouvellement de cet arrêté.

Si nous pouvons tracer un parallèle entre les pratiques d'établissements commerciaux à Calais et Lampedusa, il n'en reste pas moins que ces deux espaces conservent des spécificités importantes quant

\footnotetext{
${ }^{16}$ A des fins d'anonymisation, les prénoms et initiales des personnes interviewées sont inventées.

${ }^{17}$ Guenebeaud C., «Vivre en voisins à la frontière. Enjeux autour de la présence de migrants sans-papiers dans la ville de Calais. », Belgéo, n 2, 2015.
} 
aux configurations des contrôles frontaliers et aux possibilités d'accès aux espaces publics. L'absence de contrôles d'identités à l'extérieur du centre à Lampedusa et l'absence d'une hostilité manifeste des derniers conseils municipaux, créent une situation locale qui diffère du contexte calaisien où pouvoirs publics locaux et nationaux travaillent ensemble à soustraire les personnes migrantes à l'espace frontalier. Il en résulte que les stratégies de résistance des migrants se construisent en partie différemment en fonction de ces contextes politiques, sociaux et spatiaux particuliers.

\section{Encadré 1 : Les deux recherches. Données et méthodologie}

La recherche conduite à Lampedusa se base sur une enquête qualitative qui a combiné plusieurs sources (entretiens, observations, presse écrite, vidéos) et qui mobilise donc un ensemble de données différentes (notes d'observation, discours des enquêtés, enregistrements audiovisuels, littérature grise et rapports, etc.). Elle a été menée sur 2 ans (2013-2014) et a impliqué trois séjours sur place, deux fois pendant la saison estivale et une fois à la fin de l'hiver. Les entretiens (43 au total, d'une durée variable allant de 40 minutes à deux heures) ont permis de recueillir la parole de migrants (en 2013), d'habitants de l'île et de professionnels de l'humanitaire (en 2014). Les observations et le partage de moments ordinaires (repas, cérémonies religieuses, réunions publiques, discussions) ont permis de pénétrer progressivement l'univers de sens des interlocuteurs, notamment autour des protestations. Cette recherche a été prolongée entre avril et juin 2018, lors d'un terrain supplémentaire portant plus précisément sur l'ordinaire du contournement des procédures d'enfermement administratif et d'asile, et sur la crise de l'état de droit (52 entretiens, d'une durée allant de 50 minutes à une heure trente).

Concernant Calais, l'article se fonde sur une partie d'un travail de doctorat qui analyse de façon multiscalaire les processus de frontiérisation et d'altérisation à l'œuvre dans la ville. Dans son ensemble, l'enquête de terrain menée entre 2010 et 2014 se compose de 90 entretiens, plusieurs mois d'observations et une collecte de sources écrites. Les entretiens ont été conduits en 2013 et 2014 avec des acteurs des contrôles migratoires et des acteurs locaux : institutions politiques et administratives, services de police, transporteurs maritimes et routiers, employés du port et d'Eurotunnel, collectifs de riverains, commerçants, associations de soutien aux migrants et personnes migrantes. Ces matériaux ont été complétés par une revue de la presse locale depuis le début des années 2000 et une analyse des sources écrites produites par les acteurs interviewés. Ils sont enrichis par plusieurs mois d'observation menés pendant l'hiver et le printemps 2010 en vivant 
au quotidien dans des squats et des campements du centre-ville de Calais.

\section{Agir par et avec le corps : résistances adaptatives aux frontières}

Loin de jouer le rôle de simple toile de fond, les contextes frontaliers que l'on vient de décrire influencent la manière dont le corps des personnes en migration peut devenir un outil de résistance contre une frontière biopolitique qui confine, bloque, trie, expulse. En effet, ces personnes font preuve, de différentes façons, de leur capacité adaptative en agissant par et avec leur corps. Il s'agit ici de documenter ces différents usages du corps en tant qu'instrument du passage, en considérant d'une part leur variété (de la subversion de l'ordre public au camouflage), mais aussi leur capacité à faire émerger une figure du migrant en tant qu'agent politique ${ }^{18}$, se détachant de la vision agambienne de la victime d'un pouvoir d'exception, réduit à sa « vie nue $»^{19}$.

\subsection{Le corps souffrant}

Le fonctionnement de Calais et Lampedusa en tant que frontières a des conséquences concrètes sur la vie des migrants, tant du point de vue administratif que du point de vue psychologique et sanitaire.

L'incompréhension autour du maintien en rétention, par exemple, crée des situations de stress extrêmement difficiles à gérer, et l'incertitude prolongée quant à la procédure dont la personne fait l'objet est à l'origine d'une angoisse et d'un sentiment de détresse qui peuvent atteindre des niveaux d'une gravité extrême, comme le témoignent les suicides qui ont scandé l'histoire récente de Lampedusa (le dernier en date a eu lieu le 5 janvier 2018). Plus souvent, les personnes enfermées ont le sentiment de n'avoir rien à perdre : ainsi l'attente indéterminée au sein de la structure et l'incertitude sur son sort poussent de nombreuses personnes à commettre des actes d'automutilation (comme l'ingestion de lames de rasoir) ou à infliger des blessures au corps d'autrui (comme des coups de couteau) dans le but de provoquer l'urgence du transfert : «Je suis appelée régulièrement à intervenir en urgence à la suite de l'ingestion d'objets coupants, tels des lames ou des clous. Dans ces situations, il faut opérer, et ici il n'y a ni la structure, ni le personnel, ni les outils pour le faire. La personne doit être hélitransportée dans un hôpital, le plus souvent à Palerme. C'est une tentative désespérée de fuir,

\footnotetext{
${ }^{18}$ Rygiel R., « Bordering solidarities: migrant activism and the politics of movement and camps at Calais », Citizenship Studies, vol. 15, n 1, 2011, p. 1-19 ; De Genova N. (Ed.), The borders of 'Europe', Duke University Press, 2017 ; Ataç I., Rygiel K. et Stierl M. « Introduction: The Contentious Politics of Refugee and Migrant Protest and Solidarity Movements: Remaking Citizenship from the Margins », Citizenship Studies, vol. 20, n 5, 2011, p. 527-544.

${ }^{19}$ Giorgio Agamben (1997), Homo sacer. Le pouvoir souverain et la vie nue, Paris, Seuil.
} 
de quitter enfin les lieux. Ces personnes n'en peuvent plus, ils veulent partir cô̂te que coûte » (A., médecin bénévole, avril 2018).Le caractère « désespéré » souvent accordé à ce type de pratiques par le personnel intervenant au sein du hotspot ne saurait leur enlever tout caractère politico-stratégique en ce qu'elles témoignent avant tout d'une volonté de rupture dans l'espace-temps de l'enfermement, d'une initiative personnelle ou collective finalisée à la sortie d'une situation subie. C'est donc le corps souffrant, malade, blessé, agonisant ou exposé ${ }^{20}$, qui est utilisé comme arme pour réussir à traverser la frontière qui sépare Lampedusa de l'Italie continentale.

Ces stratégies sont moins présentes à Calais car se blesser ne conduit pas à un transfert vers l'Angleterre. Cependant, des pratiques de mise en danger des corps, comme des grèves de la faim, sont quand même ponctuellement utilisées pour faire pression sur les pouvoirs publics britanniques et français.

\subsection{Le corps dissimulé}

Ce besoin de tenter le tout pour le tout afin de quitter l'île ou le nord de la France, se traduit également, à Calais comme à Lampedusa, par d'autres formes de mobilisation du corps qui passent notamment par des pratiques de dissimulation. Une de ces stratégies de qui se retrouve largement aux frontières européennes consiste à se brûler les empreintes. Cette pratique est intimement liée à des choix effectués au niveau européen au tournant des années 2000 et en particulier, la mise en place en 2004 du système d'Eurodac, une base de données qui contient les empreintes à la fois des demandeurs d'asile, des réfugiés et des personnes interpellées lors d'un franchissement illégal d'une frontière. Le système Eurodac permet de rendre effectifs les Accords de Dublin II signés en 2003 (devenus les Accords de Dublin III en 2013), qui décident de la responsabilité d'un seul État membre dans la procédure de demande d'asile : l'Etat où il existe des traces d'entrée ou de séjour de la personne. A l'appui de l'enregistrement des migrants dans la borne Eurodac à leur arrivée en Europe, ce système permet ainsi aux pays d'Europe du Nord de renvoyer de nombreuses personnes vers le sud de l'Europe et notamment vers l'Italie.

Les personnes migrantes qui arrivent par Lampedusa, pour avoir une chance d'obtenir l'asile et de vivre dans le pays européen de leur choix, doivent donc échapper à la prise d'empreintes. Sans empreintes enregistrées dans Eurodac, elles ne pourront pas, une fois en Angleterre, en France ou en

\footnotetext{
${ }^{20}$ Fassin D., «Le corps exposé : Essai d'économie morale de l'illégitimité », in Fassin D. et Memmi D., Le gouvernement des corps. Paris, Éditions de l'École des hautes études en sciences sociales, 2004, en ligne : $<$ http://books.openedition.org/editionsehess/1499
} 
Allemagne, être ré-expulsées vers l'Italie. Elles développent donc diverses stratégies individuelles et collectives de contournement, notamment celle consistant à se brûler les bouts des doigts pour rendre non-identifiables ses empreintes ou encore celle de refuser, de manière réitérée et collective, de les enregistrer lors du passage devant la police au sein du hotspot ${ }^{21}$. Le corps dissimulé est alors à son tour un instrument de lutte par l'effacement des preuves corporelles de son identité et/ou de son passage sur le territoire d'un Etat dans lequel la personne ne souhaite pas mettre un terme à son voyage.

A la frontière franco-britannique, dans ce même contexte des accords de Dublin et de la mise en place d'Eurodac, les personnes migrantes mettent également en œuvre des tactiques de résistance à l'identification policière. L'extrait de carnet de terrain qui suit souligne la circulation de ces savoirfaire du contournement :

"Le feu crépite. Nous sommes assis dans "l'Egyptian squat 》, au premier étage du bâtiment à moitié détruit. Il manque une cloison; cela nous ouvre la vue sur le grand terrain vague. Le soleil se couche. Tout le monde est là : Adil L. Mahmoud A., Sam F., Malik R., Hélène D. et d'autres. Sam et Mahmoud racontent des histoires d'arrestation. "No, no, not like that 》 Mahmoud donne des conseils à Hélène qui est en train de brûler ses empreintes avec une barre de métal placée dans le feu. "Do it just when it's red ». "I know how to do ». C'est le premier soir que je vois Hélène ici. Adil l'aide à remettre la barre dans le feu. Puis il raconte à son tour une garde à vue.» ${ }^{22}$.

A Calais, les services de l'identité judiciaire et de l'unité judiciaire de la PAF travaillent en collaboration avec les services de la préfecture durant le temps de la garde à vue ou de la retenue. Le service de l'identité judiciaire relève les empreintes. Le rôle de l'unité judiciaire est d'obtenir des informations sur la situation de la personne grâce à des auditions. Les services de la préfecture reçoivent les dossiers et prennent les décisions de relâcher la personne ou de notifier un arrêté de reconduite à la frontière. Pour ce faire, ils consultent la borne EURODAC. Si elle est positive et qu'elle révèle que la personne a été enregistrée précédemment dans un autre état européen, ils vont opter pour une "réadmission Dublin » dans ce pays. Si elle est négative, ils vont envisager une reconduite à la frontière vers le pays d'origine ou vers un pays européen en fonction des informations récoltées dans les auditions ${ }^{23}$. Toute l'action des services de police est tournée vers cet objectif

\footnotetext{
${ }^{21}$ Lendaro A., « Nothing to Lose: The Power of Subtle Forms of Resistance in an Immigration Detention Centre », in Polese A., Russo A., Strazzari F., (Ed.), The good, the bad and the ugly. Exploring boundaries between the informal, the criminal and the immoral, Basingstoke, Palgrave Macmillan.

${ }^{22}$ Carnet de terrain, 14 mars 2010.

${ }^{23}$ Wlodarczyk M., La souveraineté de l'État à l'épreuve de la construction européenne: analyse sociologique de la stratégie étatique en matière d'éloignement forcé des étrangers, Paris, EHESS, 2011.
} 
d'identification. Se brûler les empreintes pour les rendre impossibles à lire dans Eurodac revient à ne pas faire parler son corps : il s'agit d'une des façons d'entraver les procédures qui conduisent à l'identification et à l'expulsion.

\subsection{Le corps collectif perturbateur de l'ordre public}

Ce répertoire d'action qui a trait à la dissimulation est une stratégie qui coexiste avec celle d'un corps collectif qui, inversement, perturbe l'ordre public par sa présence visible. C'est le cas en septembre 2013 à Calais où, suite à des expulsions de squats, des Syriens occupent une passerelle du port, puis s'installent sur un campement très visible à l'entrée du centre-ville, en face du lieu de distribution des repas. Après de nombreuses tractations entre la région, la mairie et la préfecture, ce campement, qui entre-temps s'est composé d'environ 300 personnes de différentes nationalités, est évacué le 28 mai 2014. Le même jour, les personnes expulsées occupent le lieu de distribution des repas situé juste en face, et les policiers échouent à les déloger. Ceux-ci essayent de forcer le passage du grillage qui entoure l'espace à plusieurs reprises, mais sans succès. L'opération policière prend fin et l'occupation se met en place. Dans les entretiens menés avec les bénévoles et les militants associatifs, plusieurs d'entre eux décrivent cette période comme une première mobilisation sur un même lieu de personnes de différentes nationalités, notamment des Syriens, des Afghans, des Érythréens, des Éthiopiens, des Soudanais, des Égyptiens et des Pakistanais. Ces derniers font le choix d'une mobilisation visible, ancrée dans le centre-ville de Calais et refusent de rejoindre les campements dans les bois en périphérie. Ils rédigent des banderoles qu'ils placent à l'extérieur du lieu le long de la route dont « We are on hunger strike until the British and French authorities solve our problems » et « We are victims of the European injustice ». La mobilisation, qui prend fin avec une expulsion policière le 2 juillet 2014, est marquante de par cette transformation du lieu de restauration en un espace de lutte et de visibilité collective.

A Lampedusa, des tentatives de provoquer l'alerte des institutions, des forces de l'ordre, du corps médical, ou des opérateurs de la structure, sont également faites via la perturbation de l'ordre public. Ainsi, dans le but d'induire une accélération dans les procédures de transfert, des groupes suffisamment nombreux peuvent se saisir de l'opportunité résidant dans le refus collectif d'enregistrer les empreintes, pour organiser des marches, manifestations, et occupations de l'espace public afin de dénoncer les conditions de détention et revendiquer le droit à la mobilité. En effet, même si un portail constamment surveillé par les forces de l'ordre sépare l'espace de confinement du dehors, des 
ouvertures dans les grillages latéraux permettent aux pensionnaires les moins craintifs et en bon état de santé de sortir. Cela étant par ailleurs un procédé connu par l'ensemble des forces de l'ordre et des habitants. Tel a été le contexte de la mobilisation dite des Erythréens de $2013^{24}$ où le corps collectif qui réussit à subvertir l'ordre public devient une arme pour faire pression sur les élus locaux et les pouvoirs publics : «Nous n'avons rien à perdre. Nous sommes enfermés depuis plusieurs semaines dans le centre, plus de mille personnes pour deux toilettes ! Surtout, nous voulons quitter l'Italie sans enregistrer nos empreintes : moi, j'aimerais rejoindre ma famille en Suède (D., Erythréen, juillet 2013). Les manifestations et les occupations de la place publique alors que la saison touristique battait son plein, ont provoqué le mécontentement d'une grande partie de la population locale, ce qui a joué en faveur des personnes mobilisées et entrainé leur transfert vers l'Italie continentale.

Loin d'être antithétiques, ces stratégies de mise en visibilité ou de dissimulation du corps constituent plusieurs versants des résistances migrantes au contrôle policier. A Lampedusa, il s'agit pour les personnes migrantes avant tout de quitter l'île et d'éviter une toute première identification biométrique en Italie, potentiellement comparable avec une autre trace de soi enregistrée ailleurs en Europe. A Calais, cet enjeu se double d'un autre : éviter l'expulsion et donc en amont l'interpellation dans les espaces publics. Cette différence conduit à une multiplication des stratégies de transformation de son corps plus vaste dans la situation calaisienne.

\subsection{Le corps normé}

L'identification policière des personnes migrantes dans les espaces publics de Calais passe par une séparation et une hiérarchisation des corps libres de circuler de ceux qui vont être entravés dans leur mobilité. Cette sélection des «corps-frontière » ${ }^{25}$ se matérialise par un ensemble de critères basés sur la couleur de peau, les vêtements, les lieux occupés ou encore les comportements adoptés dans les espaces publics. Ces critères ciblent en particulier, selon des assignations ethnoraciales, de classe et de genre, les jeunes hommes seuls et racisés qui occupent certains espaces de la ville ${ }^{26}$.

A Calais, comme dans différents espaces où est mise en œuvre cette sélection policière, une des stratégies qui est développée par les personnes migrantes est de transformer son corps pour se rapprocher d'un corps normé : blanc, européen, bourgeois. Ce corps est « insoupçonnable » dans les

\footnotetext{
${ }^{24}$ Lendaro A., «'No finger print!': Les mobilisations des migrants à Lampedusa, ou quand l'espace compte », L'Espace Politique [En ligne], vol. $25 \mathrm{n}^{\circ}$ 1, 2015, URL : http://journals.openedition.org/espacepolitique/3348.

${ }^{25}$ Guénif-Souilamas N., «Le corps-frontière, traces et trajets postcoloniaux », in Ruptures postcoloniales, Paris, La Découverte, 2010, p. 217-229

${ }^{26}$ Camille Guenebeaud, 2016, op. cit.
} 
espaces publics, transparent à l'identification policière, capable de franchir les frontières sans risquer de faire l'expérience de leur matérialité mortifère. Dans ce contexte, se rapprocher du corps normé en passant pour un Calaisien ou pour un touriste européen sont autant de manières pour une personne migrante de traverser la frontière ou d'occuper l'espace public dans l'attente de le faire, sans risquer l'expulsion. Ces pratiques sont des stratégies que nous désignons sous le terme de passing ${ }^{27}$. Elles sont mises en œuvre à Calais par des personnes migrantes dans un contexte de quadrillage policier intense du centre-ville et d'interdiction d'accès à certains lieux publics tels que les cafés. C'est le cas de Adil L. et Mahmoud M., palestiniens de respectivement 20 et 25 ans restés à Calais durant 5 mois à 1'hiver 2010 .

« Aujourd'hui, Adil L. m'a demandé à nouveau de l'accompagner au centre commercial des 4B. C'est la deuxième fois ce mois-ci. Il y a souvent des policiers à l'entrée du centre et il pense qu'en venant avec moi qui suis blanc, il risque moins l'interpellation. Son but est d'acheter régulièrement des vêtements neufs pour lui et Mahmoud A., des vêtements propres et qui fassent « français ». C'était notre objectif de l'après-midi. ${ }^{28}$

Trouver des stratégies pour acquérir des codes vestimentaires occidentaux constituent des leviers pour passer de l'identification de migrant sans-papiers à celle de touriste occidental. En ayant conscience que les policiers utilisent ces marques corporelles dans les interpellations, Adil L. tente de les déjouer dans ses déplacements. D'ailleurs, pour éviter les contrôles, il préfère souvent se déplacer seul ou avec une personne identifiée comme blanche et française qui le rapproche du corps normé.

Les stratégies du passing travaillent ainsi la dimension corporelle : choisir un style vestimentaire « occidental », adopter une certaine démarche, une façon de se tenir dans l'espace pour construire une image de touriste. Aman K., érythréen habitant de l'« Africa House », raconte en entretien qu'il sifflote pour afficher une attitude sereine dans l'espace public et qu'il regarde toujours droit devant lui ${ }^{29}$. Il adopte ainsi une démarche décidée de confiance en lui pour se fondre dans la foule et ne pas se faire

\footnotetext{
${ }^{27}$ «La notion de passing embrasse l'ensemble des tactiques dont le but est de passer pour quelqu'un'e d'autre - pour un'e membre d'un groupe auquel nous n'étions pas assigné e $\cdot s$ à la naissance. Afin que cela soit possible, il faut remplir certains critères. Il faut d'abord dresser le corps : apprendre les styles corporels appropriés (Judith Butler, Ces corps qui comptent : De la matérialité et des limites discursives du sexe, Paris, Editions Amsterdam, 2009), ce qui va souvent jusqu'à des modifications corporelles. Il faut assimiler les codes vestimentaires adéquats (Meredith Goldsmith, « Dressing, passing, and americanizing: Anzia Yezierska's sartorial fictions», Studies in American Jewish Literature, n ${ }^{\circ} 6,1997$, p. 34-45). Il faut dresser la langue (l'accent) et le langage (la façon de s'exprimer, les expressions à utiliser) » (Ian Zdanowicz, «L'architecture du passing : la place, le regard, le mouvement », Comment $S^{\prime} e n$ Sortir ?, n 2, 2015, p. 76-91)

${ }^{28}$ Carnet de terrain, 16 avril 2010

${ }^{29}$ Entretien avec Aman K., Erythréen habitant à l'« Africa House » Pagniez, réalisé le 23 mars 2010 sur un banc du parc Richelieu.
} 
remarquer. Puisque l'opération policière de contrôle consiste à « isoler [les personnes migrantes] de la foule des passants » et que, comme le souligne Stefan Le Courant, « le regard fuyant ou le brusque changement de direction attirent l'attention du policier, ce sont les "apparences" et les "manières" qu'il faut maîtriser pour composer une "apparence normale" ${ }^{30}{ }^{31}$. Le passing est alors une stratégie d'anonymat, un « art de devenir un passant $»^{32}$.

Cette stratégie déjoue les catégories par lesquelles les policiers identifient les corps soupçonnés illégaux dans les espaces publics, en même temps qu'elle a des limites. Les critères de racisation en termes de couleurs de peau laissent plus ou moins de marge de manœuvre en fonction des personnes et le passing se heurte aux marques corporelles considérées comme signifiantes dans l'identification des personnes migrantes par la société d'accueil.

A Lampedusa, l'absence de contrôle d'identité à l'extérieur de l'hotspot ne veut pas dire que le passing perd tout intérêt. Dans un contexte où l'accès à certains services (change de devises étrangères à la banque, accès payant à un Internet point) est restreint ${ }^{33}$, tout comme à Calais l'accès à certains cafés, être accompagné par un touriste ou un habitant de l'île permet d'accéder à certains espaces. Lorsque la proximité avec le corps normé ne suffit pas, c'est l'emprunt de l'identité d'autrui qui permet l'accès aux services : ainsi, il nous estarrivé d'effectuer les opérations de change à la banque à notre nom, ou encore de fournir nos documents d'identité pour pouvoir lancer une session d'utilisation d'un ordinateur connecté dans un Internat point. Néanmoins, en termes de stratégies de contournement de l'identification policière, le passing est bien moins utilisé à Lampedusa car la police n'interpelle pas dans les espaces publics. C'est donc une stratégie toujours en mouvement, jamais achevée et qui prend sens dans un contexte de normes corporelles, spatiales, et juridiques particulier ${ }^{34}$.

\section{$3 \quad$ Un droit à la subversion ?}

\footnotetext{
${ }^{30}$ Goffman E., La mise en scène de la vie quotidienne 2: Les relations en public, Paris, Ed. de Minuit, 1996.

${ }^{31}$ Le Courant S., «La ville des sans-papiers: Frontières mouvantes et gouvernement des marges », L'Homme, $\mathrm{n}^{\circ}$ 219-220, 2016, p. 209-232.

${ }^{32}$ Zdanowicz I., 2015, op. cit.

${ }^{33}$ Les gérantes d'un des deux magasins offrant un accès payant à des ordinateurs connectés à Internet sur l'île refusaient le service aux migrants de l'hotspot, sous prétexte de devoir vérifier leur identité : or, l'accès était refusé à ces personnes y compris en présence d'un passeport ou d'un document d'identité en cours de validité, puisque les propriétaires demandaient, de façon discrétionnaire et abusive, un permis de séjour. Pour la même raison, le change d'une devise étrangère à la banque se révèle une mission impossible pour les migrants de Lampedusa : si pour un italien ou européen, le seul document d'identité en cours de validité suffit, ces étrangers en transit se doivent de présenter les preuves de la légalité de leur séjour.

${ }^{34}$ Zdanowicz I., 2015, op. cit.
} 
La comparaison de ces deux études de cas fait émerger avant tout la capacité d'agir, individuellement et collectivement, de migrants illégalisés qui tentent de résister à un système de contrôle qui les confine, les éloigne des lieux publics où ils sont indésirables, et les expulse. Face à une frontière biopolitique qui s'appuie sur des choix politiques et des mesures de traçage souvent en contradiction avec les références législatives nationales et internationales en matière de droits fondamentaux, ces personnes font la preuve d'une volonté de contourner les entraves à leur mobilité qui les transforme en acteurs politiques ${ }^{35}$. Puisque le droit à la mobilité, à la santé, à la protection, à la vie familiale (entre autres) est nié, les actes de résistances par le corps varient de façon proportionnelle à la diversité des formes que le contrôle et la privation des libertés peuvent prendre aux frontières de l'UE étudiées. Ladite «crise des réfugiés » ${ }^{36}$ a rendu visible auprès du grand public l'intensité des efforts des gouvernements européens de contrôler, protéger, et finalement fermer les frontières internes et externes de l'UE face à des centaines de milliers de personnes exerçant leur droit à la mobilité transnationale. La comparaison proposée par cette contribution a quant à elle focalisé l'analyse sur les stratégies de résistance par lesquelles se manifeste l'autonomie de la migration, qui, en réponse à la surveillance accrue des frontières, prend forme dans le sabotage des procédures d'identification, dans la subversion de l'ordre public, dans la dissimulation de son identité, dans la violence stratégique infligée à son propre corps.

Comment considérer ces actes de résistance, parfois violents, sinon par l'auto-reconnaissance d'un droit à la subversion face à un système profondément inégalitaire ${ }^{37}$ ?

Le point de vue conventionnel des sociétés libérales est de considérer que l'Etat possède le droit inaliénable de contrôler l'immigration et de décréter l'appartenance d'individus et de groupes à la communauté nationale : autrement dit, le pouvoir étatique a le droit d'accepter ou non des étrangers sur son sols, et de déterminer les conditions légales de leur entrée et de leur séjour. Ce pouvoir de choisir est néanmoins (théoriquement) limité par les personnes se réclamant du droit d'asile et venant chercher protection. Droit d'asile que les volontés nationales ne cessent d'attaquer.

A la lumière de ce constat et pour conclure, nous défendons l'idée que, face aux migrations contemporaines et à la multiplicité de leurs raisons (que nous refusons par ailleurs de hiérarchiser), un droit à la mobilité transfrontalière mérite d'être défendu, y compris par le biais de pratiques de

\footnotetext{
${ }^{35}$ Isin E. F., et Nielsen G. M., Acts of Citizenship, London, Zed Books, 2008.

${ }^{36}$ Pour une interprétation critique de la formule de « crise de réfugié », voir De Genova N., 2018, op. cit.

${ }^{37}$ Carens J., The ethics of immigration, Oxford University Press, 2013.
} 
résistance qui transgressent la loi ou les procédures administratives, et perturbent parfois l'ordre public.

Par le biais de ces luttes pour la mobilité, une autre idée de société est promue, et une autre figure du « migrant» se fait protagoniste de sa défense : en désobéissant ${ }^{38}$, en transgressant les injonctions de l'Etat, en résistant y compris par son corps au régime de la frontière biopolitique, les personnes migrantes à Lampedusa et à Calais montrent comment elles négocient leur identité en tant que « sujets » ${ }^{39}$, expérimentant des façons d' «être politique » ${ }^{40}$. Et malgré la variété des formes que peuvent prendre ces résistances et leur adaptabilité aux contextes de Lampedusa et de Calais, ces actes sont une critique concrète, corporelle, de politiques migratoires considérées injustes. Il s'agit là d'une critique en actes d'un monde social se fondant sur une répartition inégalitaire de droits fondamentaux : leur demande de mobilité par-delà toute origine nationale, est une preuve de la capacité à imaginer une société à venir dans laquelle l'effectivité de ce droit ne dépendra pas d'un statut hérité à la naissance ${ }^{41}$.

\footnotetext{
${ }^{38}$ Gros F., Désobéir, Paris, Alban Michel, 2017.

${ }^{39}$ Barbero I., « Expanding acts of citizenship : the struggles of Sinpapeles Migrants », Social and Legal Studies, vol. 4, $\mathrm{n}^{\circ} 21,2012$, p. 529-547.

${ }^{40}$ Isin E. F., Being political: genealogies of citizenship. Univeristy of Minnesota Press, 2002.

${ }^{41}$ Schachar A., The Birthright lottery, Harvard University Press, 2009.
} 